\title{
Study Of Common Frequency Jamming Resistance For Airborne Radar Based On Polarization Information
}

\author{
Bo Lv \\ Department of Electronic and Optical Engineering \\ Ordnance Engineering College \\ Shijiazhuang, China \\ e-mail:1vbo2000@163.com \\ Siyang Liang \\ Department of Electronic and Optical Engineering \\ Ordnance Engineering College \\ Shijiazhuang, China \\ e-mail: Liangsy@126.com
}

\author{
Juntao Ma \\ Department of Electronic and Optical Engineering \\ Ordnance Engineering College \\ Shijiazhuang, China \\ e-mail: taotaoma@126.com \\ Lin Shi \\ Department of Electronic and Optical Engineering \\ Ordnance Engineering College \\ Shijiazhuang, China \\ e-mail: dalin1985@163.com
}

\begin{abstract}
Aiming at the problem of common-frequency jamming between airborne radars of same model, oblique projection polarization filtering technique was introduced into common frequency jamming countermeasure. The basic principle of oblique projection polarization filtering was analyzed; the anti-interference system's construction scheme and implementation steps for airborne radar were presented. Oblique projection polarization filtering method does not need signal and interference with orthogonal polarization, as long as they are difference, this method can completely suppress common frequency interference, and keep the signal amplitude and phase property, so it has extensive engineering application prospects. Computer simulation all proves the conclusion.
\end{abstract}

Keywords-common frequency jamming; polarization filtering; two-axis tracking; anti-jamming; formation flight

\section{INTRODUCTION}

Under the background of modern warfare, as one of the important tactics of modern air force deployment, formation flying has incomparable advantage compared with the single flight. In air combat, right formation could expand detection field, enhance the overall operational capability of the aircraft, the success rate of task, and flying economy, so it can achieve even greater air superiority when fighting in the air ${ }^{[1]}$. However, when planes are flying in information, they are facing a serious problem of common frequency interference between each other. Common interference is an often encountered problem when same types of radars are working together. Radar will be jammed by common frequency interference before finding enemy targets, so radars' detection performance will fall sharply, even completely paralyzed.

Now anti-jamming methods using for airborne radar are airspace method, time domain method, frequency domain method and code field method ${ }^{[2]}$. They suppress common frequency interference respectively from the aspects such as space, time, frequency and coding. Reference [3], [4] and [5] present that airborne radar can utilize the polarization difference between target signal and clutter to suppress clutters by using polarization array. In this paper, considering the posture shake of the airplanes affected by the air flow, which led to radar's actual state of polarization be changed, so we proposes a new common frequency interference suppression algorithm based on the polarization information processing. It should be pointed out that, in this paper, our study is suitable for airplanes using two-axis tracking system.

\section{POLARIZATION STATE ANALYSIS OF AIRBORNE RADAR USING TWO-AXIS TRACKING SYSTEM}

\section{A. Introduction of two-axis tracking system}

According to the structural form of antenna pedestal, two axis antenna pedestals can be divided into type of azimuth-elevation, type of X-Y, type of polar axis and so on ${ }^{[6]}$. For example of azimuth-elevation type, it is one of the most commonly used antenna pedestal. It based on ground plane, so it is also be called $-\mathrm{AZ}$ and longitude ceremony antenna pedestal. Shaft is vertical to the ground, called the azimuth axis; On axis perpendicular to the azimuth axis, known as the pitch axis.

Two axis stability systems in airborne radar are made up of posture sensor, main control computer, servo controller unit, precision servo mechanism, etc. Through GPS, we can get vehicle location information; by using carrier attitude sensor which is sensitive to initial attitude, we can get initial alignment signals; the inertial angular rate sensor is used as the feedback element of motor closed-loop servo control, which is sensitive to antenna platform changes in the direction of azimuth and elevation; through certain control to compensation, the motion platform can work stability. Control block diagram is shown in Fig. 1 . 


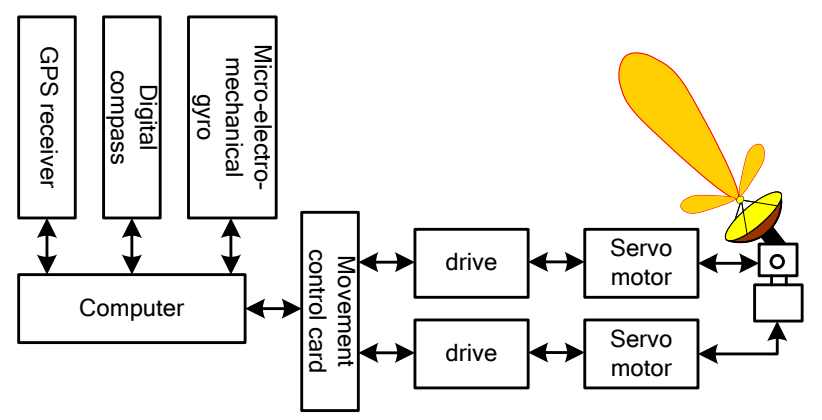

Figure 1. Diagram of airborne radar two axis tracking system

It is important to note that antenna elevation axis may roll relative to horizontal plane when the carrier attitude changes. The antenna beam will reverse rolling around its axis. Therefore, two axis stabilized tracking can only achieve stable antenna beam axis direction, cannot achieve stability of the beam. If airborne radars work in linear polarization mode, the antenna beam rolling will result in changes of transmitting and receiving polarization.

\section{B. Polarization analysis of two axis stabilized tracking}

The posture of ideal beam relative to aircraft geographic coordinate system can be expressed as the only azimuth of $\alpha$, the pitching angle of $\beta$ and the polarization angle of $\Phi$. Among them, $\alpha$ is the angle between true north and beam axis projection on the surface and clockwise is positive. $\beta$ is the beam axis and horizontal plane angle, with a positive upward. $\Phi$ is the adjusted polarization angle for the sake of polarization match, also the turning angle of the beam around its own axis, and clockwise is positive.

The airspace stabilization loop of antenna is an important part of radar antenna servo system. When the airborne radar searching and tracking targets, it can control antenna beam not to deviate from predetermined airspace in case of aircraft maneuvering.

Carrier attitude change could lead to the antenna elevation axis relative to the horizontal roll change, which is the antenna beam will reverse rolling around its axis. Therefore, two axis stabilized tracking can only achieve stable antenna beam axis direction, cannot achieve stability of the beam. Assumptions for horizontal polarization radar antenna, the antenna beam rolling will make it into a gradient polarization.

Now there are a lot of airborne radar with only two dimensional stability in azimuth and elevation, so they can only realize the stability of antenna beam axis, and cannot isolate beam rolling. Although most down-looking radar is using vertical polarization, aircraft roll will cause radar's actual polarization is not vertical polarization. So when airplane formation flight, due to the random fluctuation of body posture, radar polarization will appear random fluctuation and not identical to each other. This provides a breakthrough for the radar anti-jamming. If radar can distinguish the common frequency interference and target signal by polarization characteristics, it can realize antijamming.

\section{POLARIZATION TECHNIQUE TO SUPPRESS COMMON FREQUENCY INTERFERENCE}

At present, a lot of literatures are studying on polarization filtering technique. As long as the interference has orthogonal polarization to target signal, it can be suppressed absolutely. However, these methods are not suitable for airborne radars to suppress common frequency jamming. Because aircraft rolling although makes the radar polarization appeared difference, but it is difficult to meet the orthogonal to each other. Therefore in this paper, we propose a new method which is called oblique projection polarization filtering algorithm to suppress common frequency jamming among airborne radars.

\section{A. Oblique projection polarization filtering algorithm}

Assume that the target signal and interference are respectively as follows.

$$
\begin{aligned}
& \mathbf{S}(t)=E_{s} \cos \left(\omega_{s} t\right)\left[\begin{array}{ll}
\cos \varepsilon_{s} & \sin \varepsilon_{s} e^{\mathrm{j} \delta_{s}}
\end{array}\right]^{T}=A_{s} \mathbf{S} \\
& \mathbf{J}(t)=E_{j} \cos \left(\omega_{j} t\right)\left[\begin{array}{ll}
\cos \varepsilon_{j} & \sin \varepsilon_{j} e^{\mathrm{j} \delta_{j}}
\end{array}\right]^{T}=A_{j} \mathbf{J}
\end{aligned}
$$

Where $E_{s}$ and $E_{j}$ are the strength of the signal and interference respectively, $\omega_{s}$ and $\omega_{j}$ are the frequency of target signal and jamming, normally $E_{s}$ is less than $E_{j}$, and $\omega_{s}$ is equal to $\omega_{j}$. $\varepsilon_{s}$ and $\delta_{s}$ are target signal polarization parameters, and $\varepsilon j$ and $\delta j$ are the polarization parameters of common frequency jamming ${ }^{[7][8]}$.

$$
\begin{gathered}
\mathbf{S}=\left[\begin{array}{ll}
\cos \varepsilon_{s} & \sin \varepsilon_{s} e^{\mathrm{j} \delta_{s}}
\end{array}\right]^{T} \\
\mathbf{J}=\left[\begin{array}{ll}
\cos \varepsilon_{j} & \sin \varepsilon_{j} e^{\mathrm{j} \delta_{j}}
\end{array}\right]^{T} \\
A_{s}=E_{s} \cos \left(\omega_{s} t\right) \\
A_{j}=E_{j} \cos \left(\omega_{j} t\right)
\end{gathered}
$$

Obviously, matrix $\boldsymbol{S}$ and $\boldsymbol{J}$ are two by one column full rank matrix. We assume that the polarization state of target signal and interference are difference, so $\boldsymbol{S}$ and $\boldsymbol{J}$ column is linear independence, but don't have to be orthogonal. Therefore $[S \boldsymbol{J}]$ is also column full rank matrix, and its rank is 2 .

Along with the direction that is parallel to subspace $\langle\boldsymbol{J}\rangle$ we do oblique projection to subspace $\langle\boldsymbol{S}\rangle$, the oblique projection operator is $\boldsymbol{E}_{\boldsymbol{S J}}$.

$$
\mathbf{E}_{\mathbf{S} \mathbf{J}}=\left[\begin{array}{ll}
\mathbf{S} & \mathbf{0}
\end{array}\right]\left[\begin{array}{ll}
\mathbf{S}^{H} \mathbf{S} & \mathbf{S}^{H} \mathbf{J} \\
\mathbf{J}^{H} \mathbf{S} & \mathbf{J}^{H} \mathbf{J}
\end{array}\right]^{\dagger}\left[\begin{array}{l}
\mathbf{S}^{H} \\
\mathbf{J}^{H}
\end{array}\right]
$$

Where $\dagger$ generalized inversion and $H$ is is conjugate transpose. So, the oblique projection operator has the following properties.

$$
\mathbf{E}_{\mathbf{S J}} \mathbf{S}=\mathbf{S} \quad \mathbf{E}_{\mathbf{S J}} \mathbf{J}=0
$$

From Eq. 4, we can find that the value range of the oblique projection operator is equal to the projection space of $\langle\mathrm{S}\rangle$, and $\langle\mathrm{J}\rangle$ is a subset of the zero space of oblique projection operator. So, if we ignore the influence of random noise, the radar receiving the echo after dealing with the oblique projection operator will get the following results. 


$$
\begin{aligned}
\mathbf{y}(t) & =\mathbf{E}_{\mathrm{sJ}}\left(A_{s} \mathbf{S}+A_{j} \mathbf{J}\right) \\
& =A_{s}\left(\mathbf{E}_{\mathrm{SJ}} \mathbf{S}\right)+A_{j}\left(\mathbf{E}_{\mathrm{sJ}} \mathbf{J}\right) \\
& =A_{s} \mathbf{S} \\
& =E_{s} \cos \left(\omega_{s} t\right)\left[\begin{array}{ll}
\cos \varepsilon_{s} & \sin \varepsilon_{s} e^{j \delta_{s}}
\end{array}\right]^{T}
\end{aligned}
$$

From the Eq. 5, we draw the following conclusions. As long as the target signal and interference polarization state is different, after dealing with the oblique projection interference will be completely suppressed, but the target signal amplitude and phase will remain the same ${ }^{[9]}$. These features greatly improve its usefulness.

\section{B. Common frequency jamming suppression algorithm based on oblique projection polarization filtering}

Common frequency jamming between airborne radars are mainly direct wave interference, such as mainlobe to sidelobe jamming, sidelobe to sidelobe jamming and sidelobe to mainlobe jamming. The direct wave jamming amplitude is much larger than the target echo, so how to restrict the common frequency direct wave jamming between formation flying airborne radars becomes the key.

For the common frequency direct wave jamming, we can use the oblique projection polarization filtering algorithm to suppress it. When a aircraft is in flight process, regardless of its attitude changing, we can get its polarization state in real time. When facing common frequency jamming from other aircrafts, we can using Kalman filtering technique to access polarization parameters of jamming, then by using the oblique projection polarization filtering technique, we can easily realize the suppression of common frequency direct wave jamming. Therefore, we can build the following countermeasures system that is shown in Fig .1, its implementation steps are as follows.

Step one, to obtain local radar's polarization parameters.

Step two, to estimate jamming polarization parameters by using Kalman filtering technique ${ }^{[10]}$.

Step three, by using Eq. 3, oblique projection operator $E_{S J}$ is derived.

Step four, $E_{S J}$ acts on the raw echo signal, restrain the common frequency jamming and get pure target signal.

Oblique projection polarization filtering technique is obviously superior to the traditional polarization filtering technique, because it does not need interference and signal polarization completely orthogonal. This new method can restrain the common frequency interference, and keep the signal amplitude and phase constant, so these features greatly improve the practicability of the new method.

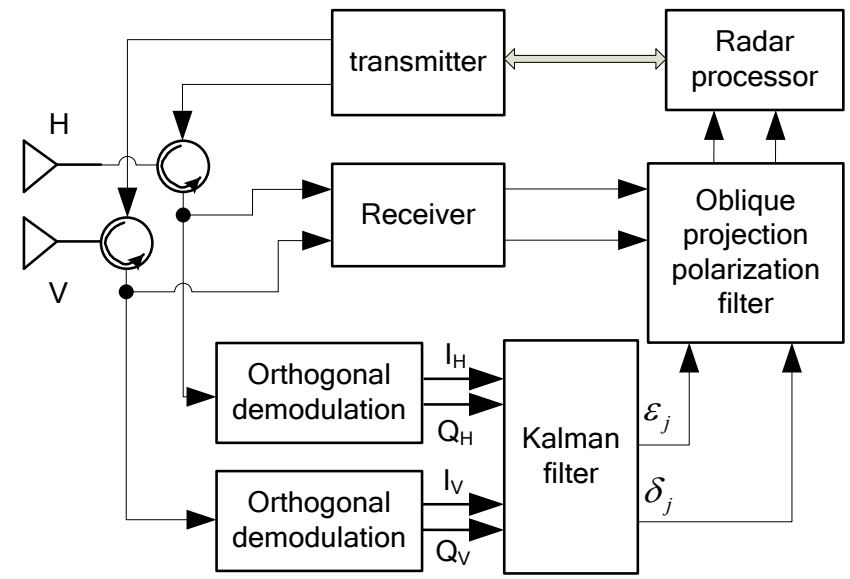

Figure 2. Principle block diagram of polarization filter to anti-jamming

\section{EXPERIMENT AND SIMULATION}

Airborne radar is facing with common frequency synchronous interference from nearby radars, and the interference is line polarization interference, and its polarization vector is $\left[\cos 80^{\circ} \sin 80^{\circ}\right]$. So $\varepsilon_{j}$ is equal to $80^{\circ}$, and $\delta_{j}$ is equal to 0 . Target signal is also line polarization. Its polarization vector is $\left[\cos 70^{\circ} \sin 70^{\circ}\right]$, so $\varepsilon_{j}$ is equal to $70^{\circ}$, and $\delta_{j}$ is equal to 0 . The common frequency interference strength is greater than the target echo. Interference signal power ratio is $20 \mathrm{~dB}$, interference noise power ratio is $30 \mathrm{~dB}$, and $\mathrm{SNR}$ is $10 \mathrm{~dB}$. Radar repeat cycle is $20 \mathrm{us}$ and pulse width is $1 \mathrm{us}$.

Using the Matlab software simulation, we get the following comparison results as show in Fig .2 and 3 .

Fig . 2 is the radar echo not using the anti-interference technique. In addition to a target echo, there is also significant common frequency interference. If send this echo to radar for processing, radar is round to be disturbed by common frequency interference inevitably, so radar detection performance will decline. In addition, by contrasting Fig .2 (a) and (b), we can find that the magnitude of vertical polarization channel is greater than horizontal channel because the polarization of interference and target echo are all close to vertical polarization.

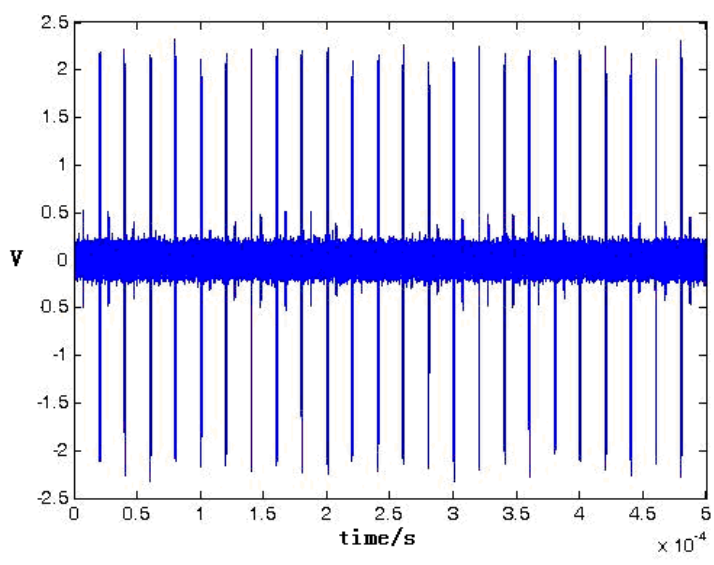

(a) Horizontal polarization channel 


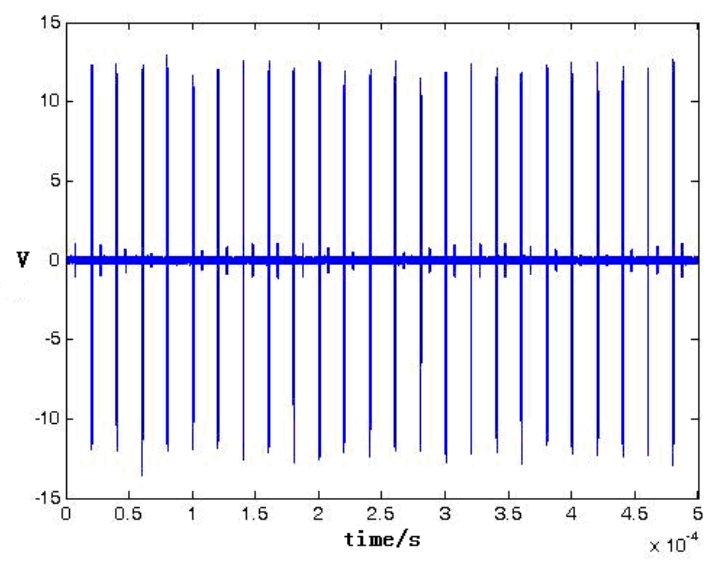

(b) Vertical polarization channel

Figure 3. Radar is facing with common frequency interference



(a) Horizontal polarization channel



(b) Vertical polarization channel

Figure 4. Output echo by using polarization filtering technique
Fig .3 gives the echo of vertical polarization channel and horizontal polarization by using the oblique projection polarization filtering technique that is put forward in this paper. Obviously, common frequency interference in two polarization channel is suppressed, leaving only real target and noise. Due to the movement of real target, radar echo pulse envelope is modulated by Doppler frequency.

By comparing the simulation results in Fig .2 and 3, the oblique projection polarization filtering technique that is put forward in this paper is effective, and it can greatly improve the detection performance of airborne radar.

\section{CONCLUSION}

Polarization domain is an effective way to suppress common frequency interference. When the planes are flying in formation, the common frequency jamming will be inevitable among them. In this paper, considering the random fluctuation of carrier aircraft using two axis stability systems, the actual state of radar polarization will vary from each other. We use the oblique projection polarization filtering technique to suppress the common frequency jamming. The new method's effect is obvious.

\section{REFERENCES}

[1] Feifei Fang, "Research of anti-collision algorithm of formation flight based on the probability," Chengdu: Master's thesis of University of Electronic Science and Technology, 2011, pp. I.

[2] Hou Minsheng, Qin Haichao, "Study on coherent accumulation against interference between radars with same frequency," Electronic Measurement Technology, Vol.31, 2008, pp. 51-53.

[3] Wu Dijun, Xu Zhenhai, Xiong Ziyuan, "Performance analysis of polarization-space-time joint domain processing for clutter suppression in airborne radars," Acta Electronica Sinica, Vol. 40, 2012, pp. 1429-1433.

[4] Zhang Guoyi, Wang Jiantao, Lu jingshuang, "Polarization suppression of local radar signal in airborne radar reconnaissance,' Systems Engineering and Electronics, Vol. 28, 2006, pp. 15161518 .

[5] Wu Dijun, Xu Zhenhai, Xiong Ziyuan, "Performance analyse on polarization-space-time adaptive filter in airborne radars," Journal of Electronics \& Information Technology, Vol. 34, 2012, pp. 2128 2134

[6] Yongzhi Zha, "Study on measurement and control technology of tracking mobile carrier stable platform," Changsha: Master's thesis of National University of Defense Technology, 2002, pp. 5-10.

[7] Zeng Qingping, Yan Shiqiang, Radar polarization technique and application of polarization information, Beijing: National Defense Industry Press, 2006, pp.1-10.

[8] Zhuang Zhaowen, Xiao Shunping, Wang Xuesong, Processing and application of radar polarization information, Beijing: National Defense Industry Press, 1999, pp.123-130.

[9] Bo Lv, "A new method to eliminate target effect in sidelobe canceller," Chinese Journal of Ratio Science, Vol. 28, 2013, pp. 547-552.

[10] Pang Bo, Pan Jian, "A study on adaptive polarization estimation method,” Moern Radar, Vol. 31, 2009, pp. 38-46. 